\title{
ON SOME GENERAL ALMOST PERIODIC OPTIMAL CONTROL PROBLEMS: LINKS WITH PERIODIC PROBLEMS AND NECESSARY CONDITIONS*
}

\author{
Denis PenNequin ${ }^{1}$
}

\begin{abstract}
In this paper, we are concerned with periodic, quasi-periodic (q.p.) and almost periodic (a.p.) Optimal Control problems. After defining these problems and setting them in an abstract setting by using Abstract Harmonic Analysis, we give some structure results of the set of solutions, and study the relations between periodic and a.p. problems. We prove for instance that for an autonomous concave problem, the a.p. problem has a solution if and only if all problems (periodic with fixed or variable period, q.p. or a.p.) have a constant solution. After that, we give some first order necessary conditions (weak Pontryagin) in the space of Harmonic Synthesis and we also give in this space an existence result.
\end{abstract}

Mathematics Subject Classification. 43A60, 49K27, 49J27.

Received September 8, 2006. Revised January 18, 2007.

Published online December 21, 2007.

\section{INTRODUCTION}

Periodic Optimal Control problems are motivated by a lot of applications (see for instance $[9,14,18,21]$ and their references). It is for instance known in industry that a periodic control can give a better production process than a static one $[17,26]$. But for these problems, even if the period is chosen, the class of periodic controls is too restrictive. For instance, the sum or the convex combinaison of two periodic functions is generally not periodic but quasi-periodic (q.p.). The natural class to study this problem is in fact the class of almost periodic (a.p.) functions which, roughly speaking, allows linear combinations and limits (in a adequate sense).

Almost periodic problems have been first studied by Da Prato and Ichikawa [11], in the particular case of stochastic linear-quadratic problems. In our paper, we just consider deterministic problems, but one of the aims is to obtain necessary conditions theorems with less restrictive assumptions on the state equation and the integrand of the functional (Sect. 4). We will also give some structure results and the conditions which ensure the fact that the values of periodic, quasi-periodic and almost periodic problems have the same value. This will be the aim of Section 3. Section 5 is devoted to an existence result. Before that, in Section 1, we introduce the different problems and we precise the assumptions in Section 2. With the language of Abstract Harmonic

\footnotetext{
Keywords and phrases. Almost Periodic Optimal Control, Periodic Optimal Control, Pontryagin theorem, Almost periodicity on groups.

* I would like to thank J. Blot and the reviewers for helpful comments.

${ }^{1}$ Laboratoire Marin MERSENNE, Université Paris 1 Panthéon-Sorbonne, Centre P.M.F., 90 rue de Tolbiac, 75647 Paris cedex 13, France; pennequi@univ-paris1.fr
} 
Analysis, it is possible to set all our different problems in a common formalism. But first, we recall some facts on a.p. and q.p. functions (see for instance $[2,5,8,10,13,20]$ ).

$\mathrm{AP}^{0}\left(\mathbb{R}, \mathbb{K}^{n}\right)(\mathbb{K}=\mathbb{R}$ or $\mathbb{C})$ is the space of (Bohr)-almost periodic functions. One of the definition consists in saying that $f \in \operatorname{AP}^{0}\left(\mathbb{R}, \mathbb{K}^{n}\right)$ if and only if, for any $\varepsilon>0$, we can find an integer $k \geq 1$ and continuous periodic functions $f_{1}, \ldots, f_{k}$ such that:

$$
\sup _{x \in \mathbb{R}}\left|f(x)-\sum_{i=1}^{k} f_{i}(x)\right|<\varepsilon .
$$

This space, endowed with the uniform-norm, is a Banach space. We recall that each $f \in \operatorname{AP}^{0}\left(\mathbb{R}, \mathbb{K}^{n}\right)$ admits a mean value:

$$
\mathcal{M}\{f\}:=\lim _{T \rightarrow+\infty} \frac{1}{T} \int_{0}^{T} f(t) \mathrm{d} t
$$

and that we can develop them as a Fourier-Bohr expansion:

$$
f \sim \sum_{\lambda \in \mathbb{R}} a_{\lambda} e_{\lambda}
$$

where $e_{\lambda}:=\left[t \mapsto \mathrm{e}^{i \lambda t}\right]$. When the $\mathbb{Z}$-module generated by $\left\{\lambda \in \mathbb{R}, a_{\lambda} \neq 0\right\}$ admits a finite basis $\omega_{1}, \ldots, \omega_{m}$, we say that $f$ is quasi-periodic with frequencies $\omega:=\left(\omega_{1}, \ldots, \omega_{m}\right)$ and we write $f \in \mathrm{QP}_{\omega}^{0}\left(\mathbb{R}, \mathbb{K}^{n}\right)$. Next, we introduce by induction the spaces $\mathrm{AP}^{k}\left(\mathbb{R}, \mathbb{K}^{n}\right)\left(\right.$ resp. $\left.\mathrm{QP}_{\omega}^{k}\left(\mathbb{R}, \mathbb{K}^{n}\right)\right): f$ is in $\mathrm{AP}^{k}\left(\mathbb{R}, \mathbb{K}^{n}\right)\left(\operatorname{resp} . \mathrm{QP}_{\omega}^{k}\left(\mathbb{R}, \mathbb{K}^{n}\right)\right)$ if $f$ is of class $C^{k}$ and if $f^{\prime}$ is in $\mathrm{AP}^{k-1}\left(\mathbb{R}, \mathbb{K}^{n}\right)$ (resp. $\mathrm{QP}_{\omega}^{k-1}\left(\mathbb{R}, \mathbb{K}^{n}\right)$ ). The completion of $\mathrm{AP}^{0}\left(\mathbb{R}, \mathbb{K}^{n}\right)$ (resp. $\mathrm{QP}_{\omega}^{0}\left(\mathbb{R}, \mathbb{K}^{n}\right)$ ) with respect to the following scalar product:

$$
\langle f, g\rangle:=\mathcal{M}\{f \cdot \bar{g}\}
$$

is denoted $\mathrm{B}^{2}\left(\mathbb{R}, \mathbb{K}^{n}\right)$ (resp. $\left.\mathrm{B}_{\omega}^{2}\left(\mathbb{R}, \mathbb{K}^{n}\right)\right)$ and is the Besicovitch space. Blot (cf. [4]) has introduced an asSobolev weak notion of derivation on these spaces. The resulting Hilbert spaces are denoted $\mathrm{B}^{1,2}\left(\mathbb{R}, \mathbb{K}^{n}\right)$ $\left(\right.$ resp. $\left.\mathrm{B}_{\omega}^{1,2}\left(\mathbb{R}, \mathbb{K}^{n}\right)\right)$.

When the module of frequencies is fixed, $\omega=\left(\omega_{1}, \ldots, \omega_{m}\right)$, we can speak of $\omega$-quasi-periodicity by using the Percival formalism [23,24] which is made precise in [6]. The mapping:

$$
\mathcal{Q}_{\omega}:=[u \longmapsto[t \mapsto u(t \omega)]]
$$

maps functions from $\mathbb{T}^{m}$ (i.e. function from $\mathbb{R}^{m}$ which are $2 \pi$-periodic in each variable) to quasi-periodic functions with module of frequencies $\omega$, it satisfies (when the derivatives exist): $\mathcal{Q}_{\omega} \circ \partial_{\omega}=\frac{\mathrm{d}}{\mathrm{d} t} \circ \mathcal{Q}_{\omega}$ where $\partial_{\omega} u:=\sum_{i=1}^{m} \omega_{i} \frac{\partial u}{\partial x_{i}}$. Moreover, this mapping is a linear isomorphism of Banach (or Hilbert) spaces in these cases:

- $\mathrm{C}^{0}\left(\mathbb{T}^{m}, \mathbb{R}^{n}\right) \longrightarrow \mathrm{QP}_{\omega}^{0}\left(\mathbb{R}, \mathbb{R}^{n}\right)$.

- $\mathrm{L}^{2}\left(\mathbb{T}^{m}, \mathbb{R}^{n}\right) \longrightarrow \mathrm{B}_{\omega}^{2}\left(\mathbb{R}, \mathbb{R}^{n}\right)$.

- $\left\{u \in \mathrm{C}^{0}\left(\mathbb{T}^{m}, \mathbb{R}^{n}\right), \exists \partial_{\omega} u \in \mathrm{C}^{0}\left(\mathbb{T}^{m}, \mathbb{R}^{n}\right)\right\}=: \mathrm{C}_{\omega}^{1}\left(\mathbb{T}^{m}, \mathbb{R}^{n}\right) \longrightarrow \mathrm{QP}_{\omega}^{1}\left(\mathbb{R}, \mathbb{R}^{n}\right)$.

- $\left\{u \in \mathrm{L}^{2}\left(\mathbb{T}^{m}, \mathbb{R}^{n}\right), \exists \partial_{\omega} u \in \mathrm{L}^{2}\left(\mathbb{T}^{m}, \mathbb{R}^{n}\right)\right\}=: \mathrm{H}_{\omega}^{1}\left(\mathbb{T}^{m}, \mathbb{R}^{n}\right) \longrightarrow \mathrm{QP}_{\omega}^{1}\left(\mathbb{R}, \mathbb{R}^{n}\right)$. 


\section{ON SOME PROBLEMS}

\subsection{Periodic problem with a fixed period}

Assuming that the function $f_{0}(., x, u)$ is $\tau$-periodic for all $(x, u) \in \mathbb{R}^{N} \times \mathbb{R}^{M}$, one of the first problems we can consider is:

$$
\left\{\begin{array}{l}
\text { Maximize } \frac{1}{\tau} \int_{0}^{\tau} f_{0}(t, x(t), u(t)) \mathrm{d} t \\
(x, u) \tau \text {-periodic } \\
\dot{x}(t)=f(t, x(t), u(t)) .
\end{array}\right.
$$

Introducing the mean operator $\mathcal{M}$, this problem can be written in the following form:

$$
\left\{\begin{array}{l}
\text { Maximize } \mathcal{M}\left\{f_{0}(t, x(t), u(t))\right\}_{t} \\
(x, u) \tau \text {-periodic } \\
\dot{x}(t)=f(t, x(t), u(t))
\end{array}\right.
$$

which we be called later $(\mathbf{F P P P}(\tau))$ (for fixed-period periodic problem with period $\tau$ ). This problem can also be considered when $f_{0}(., x, u)$ is non necessarily periodic, but such that the mean exists for any $(x, u)$ varying in an appropriate space of periodic functions.

\subsection{Periodic problem with a variable period}

Assuming that the function $f_{0}$ is autonomous (i.e. does not depend on $t$ ), the second problem we can consider is:

$$
\left\{\begin{array}{l}
\text { Maximize } \frac{1}{\tau} \int_{0}^{\tau} f_{0}(t, x(t), u(t)) \mathrm{d} t \\
(x, u) \tau \text {-periodic } \\
\dot{x}(t)=f(t, x(t), u(t))
\end{array}\right.
$$

where in fact $f_{0}(t, x(t), u(t))=f_{0}(x(t), u(t))$. Introducing the mean operator $\mathcal{M}$, this problem can be written in the following form:

$$
\left\{\begin{array}{l}
\text { Maximize } \mathcal{M}\left\{f_{0}(t, x(t), u(t))\right\}_{t} \\
\tau>0, \quad(x, u) \tau \text {-periodic } \\
\dot{x}(t)=f(t, x(t), u(t))
\end{array}\right.
$$

which we be called later (VPPP) (for variable period periodic problem). This second form will also be considered when $f_{0}$ is non autonomous.

\subsection{Quasi periodic problem with a fixed module frequencies}

Here, we are concerned with the problem, where $\omega$ is fixed and for suitable $f_{0}$ :

$$
\left\{\begin{array}{l}
\text { Maximize } \mathcal{M}\left\{f_{0}(t, x(t), u(t))\right\}_{t} \\
(x, u) \omega \text {-quasi periodic } \\
\dot{x}(t)=f(t, x(t), u(t))
\end{array}\right.
$$

By using Percival's formalism, this problem can be transformed on the torus. It can be written as:

$$
\left\{\begin{array}{l}
\text { Maximize } \int_{\mathbb{T}^{m}} F_{0}(x, X(x), U(x)) \mathrm{d} x \\
(X, U) 2 \pi \text {-periodic in each variable } \\
\partial_{\omega} X(x)=F(x, X(x), U(x))
\end{array}\right.
$$

where $F_{0}$ (resp. $F$ ) is deduced from $f_{0}$ (resp. $f$ ) applying the Percival formalism at $f_{0}(., x, u)$ for all $(x, u)$, i.e. $F_{0}(., x, u)=\mathcal{Q}_{\omega}^{-1}\left(f_{0}(., x, u)\right)$ for each $(x, u)$. This problem will be named (QPP) in the sequel. 


\subsection{Almost periodic problems}

The form of a.p. problem that we can consider on $\mathbb{R}$ is, with suitable $f_{0}$ :

$$
\left\{\begin{array}{l}
\text { Maximize } \mathcal{M}\left\{f_{0}(t, x(t), u(t))\right\}_{t} \\
(x, u) \text { almost periodic } \\
\dot{x}(t)=f(t, x(t), u(t))
\end{array}\right.
$$

which we be called later (APP) (for almost periodic problem).

\subsection{Problems in discrete time}

We could also consider discrete time problems, in the case of periodic or almost periodic problems. We recall $[7,22]$ that almost periodicity for sequences can be viewed in different equivalent ways (via formal definition, extension to the Bohr compactification of $\mathbb{Z}$, or sequence which can be interpolated by a.p. functions). One of the specific features of discrete time problems is that the periodicity is necessarily with period in $\mathbb{N}^{*}$. We do not stay more on these problems because we will include them in an abstract setting.

\subsection{Abstract almost periodic problems}

We can treat the quasi-periodic and almost periodic cases, with respect to either discrete or continuous time, in a unified approach by means of Commutative Harmonic Analysis [15,16,19,25,27,28].

So, we consider in what follows a locally compact abelian group $\mathbb{G}$ whose Haar measure will be denoted $\mu_{\mathbb{G}}$; its Bohr compactification will be denoted $\mathbb{B} \mathbb{G}$. We recall that $\mathbb{B} \mathbb{G}$ is a compact abelian group and that there exists an injection $\iota: \mathbb{G} \rightarrow \mathbb{B} \mathbb{G}$ such that $\iota(\mathbb{G})$ is dense in $\mathbb{B} \mathbb{G}$. The Haar measure of $\mathbb{B} \mathbb{G}$ will de denoted $\mu_{\mathbb{B} \mathbb{G}}$. Almost periodicity on the group $\mathbb{G}$ can be translated on functions on $\mathbb{B} \mathbb{G}$ : a function $f: \mathbb{G} \rightarrow \mathbb{R}^{N}$ is a.p. if it can be extended to a function $\hat{f} \in \mathrm{C}^{0}\left(\mathbb{B} \mathbb{G}, \mathbb{R}^{N}\right)$. Such a function is necessarily unique, and we will write it also $f$.

Now, we consider an unbounded continuous linear operator $\Theta$ defined from $\mathrm{D}^{0} \subset \mathrm{C}^{0}\left(\mathbb{B} \mathbb{G}, \mathbb{R}^{N}\right) \rightarrow \mathrm{C}^{0}\left(\mathbb{B} \mathbb{G}, \mathbb{R}^{N}\right)$ and from $\mathrm{D}^{2} \subset \mathrm{L}^{2}\left(\mathbb{B} G, \mathbb{R}^{N}\right) \rightarrow \mathrm{L}^{2}\left(\mathbb{B G}, \mathbb{R}^{N}\right)$ such that, if $x \in \mathrm{D}^{0} \cap \mathrm{D}^{2}$, the two definitions of $\Theta(x)$ are equivalent. If $\mathrm{D}^{0}\left(\right.$ resp. $\left.\mathrm{D}^{2}\right)$ is a strict subset of $\mathrm{C}^{0}\left(\mathbb{B} \mathbb{G}, \mathbb{R}^{N}\right)\left(\right.$ resp. $\left.\mathrm{L}^{2}\left(\mathbb{B} \mathbb{G}, \mathbb{R}^{N}\right)\right)$, we set:

$$
\|x\|_{\mathrm{D}^{0}}=\|x\|_{\infty}+\|\Theta(x)\|_{\infty} \quad\left(\text { resp. }\|x\|_{\mathrm{D}^{2}}=\sqrt{\|x\|_{\mathrm{L}^{2}\left(\mathbb{B G}, \mathbb{R}^{N}\right)}^{2}+\|\Theta(x)\|_{\mathrm{L}^{2}\left(\mathbb{B G}, \mathbb{R}^{N}\right)}^{2}}\right)
$$

and if $\mathrm{D}^{0}=\mathrm{C}^{0}\left(\mathbb{B} G, \mathbb{R}^{N}\right)\left(\right.$ resp. $\left.\mathrm{D}^{2}=\mathrm{L}^{2}\left(\mathbb{B} \mathbb{G}, \mathbb{R}^{N}\right)\right)$ we set $\|x\|_{\mathrm{D}^{0}}=\|x\|_{\infty}\left(\right.$ resp. $\left.\|x\|_{\mathrm{D}^{2}}=\|x\|_{\mathrm{L}^{2}\left(\mathbb{B G}, \mathbb{R}^{N}\right)}\right)$. The adjoint operator $\Theta^{*}$ will always be the adjoint with respect to the $\mathrm{L}^{2}$ duality.

We can consider the abstract problem, which will be denoted by (AAPP):

$$
\left\{\begin{array}{l}
\text { Maximize } \int_{\mathbb{B G}} f_{0}(\gamma, x(\gamma), u(\gamma)) \mathrm{d} \mu_{\mathbb{B G}}(\gamma) \\
(x, u) \in \mathrm{D}^{0} \times \mathrm{C}^{0}\left(\mathbb{B} \mathbb{G}, \mathbb{R}^{N}\right)\left(\text { or } \mathrm{D}^{2} \times \mathrm{L}^{2}\left(\mathbb{B G}, \mathbb{R}^{N}\right)\right) \\
\Theta(x)(\gamma)=f(\gamma, x(\gamma), u(\gamma)) .
\end{array}\right.
$$

Now, we explain why the preceding problems are particular cases of this one.

1. The almost periodic problem in continuous time. Here, $\mathbb{G}=\mathbb{R}$ and the operator $\Theta$ is the derivative: $\Theta(x)=\dot{x} . \mathrm{D}^{0}=\mathrm{AP}^{1}\left(\mathbb{R}, \mathbb{R}^{N}\right)$ and $\mathrm{D}^{2}=\mathrm{B}^{1,2}\left(\mathbb{R}, \mathbb{R}^{N}\right)$. Here, we have $\Theta^{*}(x)=-\dot{x}$.

2. The almost periodic problem in discrete time. Here, $\mathbb{G}=\mathbb{Z}$ and the operator $\Theta$ is the right shift: $(\Theta(x))(t)=x_{t+1}\left(\right.$ and $\left.\left(\Theta^{*}(x)\right)(t)=x_{t-1}\right)$. Here, $\mathrm{D}^{0}=\mathrm{C}^{0}\left(\mathbb{B} \mathbb{G}, \mathbb{R}^{N}\right)$ and $\mathrm{D}^{2}=\mathrm{L}^{2}\left(\mathbb{B} \mathbb{G}, \mathbb{R}^{N}\right)$.

3. The quasi periodic problem with fixed frequencies. Here, $\mathbb{G}=\mathbb{T}^{m}$ and the operator $\Theta$ is the partial derivative: $\Theta(X)=\partial_{\omega} X$ (and $\left.\Theta^{*}(X)=-\partial_{\omega} X\right)$. Here, $\mathrm{D}^{0}=\mathrm{QP}_{\omega}^{1}\left(\mathbb{R}, \mathbb{R}^{N}\right)$ and $\mathrm{D}^{2}=\mathrm{H}_{\omega}^{1}\left(\mathbb{T}^{m}, \mathbb{R}^{n}\right)$. 
In all cases $\mathbf{1}-\mathbf{3}$, the mean operator $\mathcal{M}$ satisfies the following identity:

$$
\forall \varphi \in A P\left(\mathbb{G}, \mathbb{R}^{N}\right), \quad \mathcal{M}\{\varphi\}=\int_{\mathbb{B} \mathbb{G}} \varphi(\gamma) \mathrm{d} \mu_{\mathbb{B} \mathbb{G}}(\gamma) .
$$

\section{The SETTINGS}

We will in what follows consider two cases: the regular case, and the weak case. The second one corresponds to Hilbert spaces where the Harmonic synthesis is valid.

Let us now give some details about assumptions underlying our analysis. We explain the settings for periodic problems and for abstract almost periodic problems. The cases of quasi-periodic problems and almost periodic problems will be seen as particular cases of the abstract almost periodic problem.

\subsection{Periodic problems}

Regular case. We assume that $f_{0} \in \mathrm{C}^{0}\left(\mathbb{B} \mathbb{R} \times \mathbb{R}^{N} \times \mathbb{R}^{M}, \mathbb{R}\right)$ and that $f \in \mathrm{C}^{0}\left(\mathbb{B} \mathbb{R} \times \mathbb{R}^{N} \times \mathbb{R}^{M}, \mathbb{R}^{N}\right)$. The constraints sets are in this case:

$$
\mathbf{C}(\operatorname{PPFP}(\tau)):=\left\{(x, u) \in \mathrm{C}_{\tau}^{1}\left(\mathbb{R}, \mathbb{R}^{N}\right) \times \mathrm{C}_{\tau}^{0}\left(\mathbb{R}, \mathbb{R}^{M}\right), \quad \dot{x}=f(., x, u)\right\}
$$

and

$$
\mathbf{C}(\mathbf{P P V P}):=\cup_{\tau>0} \mathbf{C}(\mathbf{P P F P}(\tau)) .
$$

Remark 2.1. Here, we do not assume that $f_{0}(., x, u)$ is periodic, but we do the assumption that $f_{0} \in \mathrm{C}^{0}(\mathbb{B} \mathbb{R} \times$ $\left.\mathbb{R}^{N} \times \mathbb{R}^{M}, \mathbb{R}\right)$. It is equivalent to say that $f_{0}$ is a.p. uniformly with respect to its second and third argument. It permits us to deduce the fact that, if $(x, u)$ is a.p. (and consequently if it is periodic), then $t \mapsto f_{0}(t, x(t), u(t)$ ) is a.p., and so the mean in the functional exists.

Weak case. The functions $f$ and $f_{0}$ are Caratheodory, $f(t, .,$.$) is Gâteaux-differentiable for any t$, the Gâteaux differentials $D_{x}^{G} f$ and $D_{u}^{G} f$ are Caratheodory, and these functions satisfy for all $(t, x, u) \in \mathbb{R} \times \mathbb{R}^{N} \times \mathbb{R}^{M}$ :

$$
\begin{gathered}
\left|f_{0}(t, x, u)\right| \leq c\left(|x|^{2}+|u|^{2}\right)+d(t) \\
\forall \varphi \in\left\{f, D_{x}^{G} f, D_{u}^{G} f\right\}, \quad|\varphi(t, x, u)| \leq c(|x|+|u|)+d(t)
\end{gathered}
$$

with $c>0$ and $d \in L^{1}\left(\mathbb{B R}, \mathbb{R}^{+}\right)$.

The constraints sets are in this case:

$$
\mathbf{C}(\operatorname{PPFP}(\tau)):=\left\{(x, u) \in \mathrm{H}_{\tau}^{1}\left(\mathbb{R}, \mathbb{R}^{N}\right) \times \mathrm{L}_{\tau}^{2}\left(\mathbb{R}, \mathbb{R}^{M}\right), \quad \dot{x}=f(., x, u)\right\}
$$

and

$$
\mathbf{C}(\mathbf{P P V P}):=\cup_{\tau>0} \mathbf{C}(\mathbf{P P F P}(\tau))
$$

where $\mathrm{H}_{\tau}^{1}\left(\mathbb{R}, \mathbb{R}^{N}\right)\left(\operatorname{resp} . \mathrm{L}_{\tau}^{2}\left(\mathbb{R}, \mathbb{R}^{M}\right)\right)$ is the space of functions in $\mathrm{H}_{\text {loc }}^{1}\left(\mathbb{R}, \mathbb{R}^{N}\right)\left(\right.$ resp. $\left.\mathrm{L}_{\text {loc }}^{2}\left(\mathbb{R}, \mathbb{R}^{M}\right)\right)$ which are $\tau$-periodic.

\subsection{Abstract almost periodic problems}

Regular case. We assume that $f_{0} \in \mathrm{C}^{0}\left(\mathbb{B} \mathbb{G} \times \mathbb{R}^{N} \times \mathbb{R}^{M}, \mathbb{R}\right)$ and that $f \in \mathrm{C}^{0}\left(\mathbb{B} \mathbb{G} \times \mathbb{R}^{N} \times \mathbb{R}^{M}, \mathbb{R}^{N}\right)$. The constraint set is in this case:

$$
\mathbf{C}(\mathbf{A A P P}):=\left\{(x, u) \in \mathrm{D}^{0} \times \mathrm{C}^{0}\left(\mathbb{B} \mathbb{G}, \mathbb{R}^{M}\right), \quad \Theta(x)=f(., x, u)\right\} .
$$


Weak case. The functions $f$ and $f_{0}$ are Caratheodory, $f(\gamma, .,$.$) is Gâteaux-differentiable for any \gamma$, the Gâteaux differentials $D_{x}^{G} f$ and $D_{u}^{G} f$ are Caratheodory, and these functions satisfy for all $(\gamma, x, u) \in \mathbb{B} \mathbb{G} \times \mathbb{R}^{N} \times \mathbb{R}^{M}$ :

with $c>0$ and $d \in L^{1}\left(\mathbb{B} \mathbb{G}, \mathbb{R}^{+}\right)$.

$$
\begin{gathered}
\left|f_{0}(\gamma, x, u)\right| \leq c\left(|x|^{2}+|u|^{2}\right)+d(\gamma) \\
\forall \varphi \in\left\{f, D_{x}^{G} f, D_{u}^{G} f\right\}, \quad|\varphi(\gamma, x, u)| \leq c(|x|+|u|)+d(\gamma)
\end{gathered}
$$

The constraint set is in this case:

$$
\mathbf{C}(\mathbf{A A P P}):=\left\{(x, u) \in \mathrm{D}^{2} \times \mathrm{L}^{2}\left(\mathbb{B} G, \mathbb{R}^{M}\right), \quad \Theta(x)=f(., x, u)\right\} .
$$

\section{LiNKS BETWEEN THE PERIODIC PROBLEMS AND THE ALMOST PERIODIC PROBLEM IN CONTINUOUS TIME}

The aim of this section is to study, in a case of linear-concave problems, the link between the problems $(\mathbf{F P P P}(\tau)),(\mathbf{V P P P}),(\mathbf{A P P})$, and to obtain some structure results.

We first, as an introduction, give an explicit example. Let us consider the q.p. (but not periodic) function $g$ defined by:

We consider the following control problem:

$$
g(t):=\cos (t)+\cos (\pi t) .
$$

$$
\left\{\begin{array}{l}
\text { Maximize } J(x, u):=\mathcal{M}\left\{-(u-g)^{2}\right\} \\
\dot{x}=u
\end{array}\right.
$$

It is straightforward to identify the solutions of periodic problems with fixed period $\tau$ by using the Fourier-Bohr expansions:

(1) when $\tau /(2 \pi) \in \mathbb{N}^{*}$, the solution is $\left(x_{0}, \dot{x_{0}}\right)$ with $x_{0}(t):=\sin (t)$ and the value of the problem is $-1 / 2$;

(2) when $\tau / 2 \in \mathbb{N}^{*}$, the solution is $\left(x_{1}, \dot{x_{1}}\right)$ with $x_{1}(t):=\sin (\pi t) / \pi$ and the value of the problem is $-1 / 2$;

(3) in other cases, the solution is $(0,0)$ and the value of the problem is -1 .

From that, we deduce that the value of the variable-period problem is $-1 / 2$. Now, if we consider the q.p. problems, we can take the convex combinaison of $x_{0}$ and $x_{1}$ : by putting $x_{\alpha}:=(1-\alpha) x_{0}+\alpha x_{1}$, we see by a simple calculation that:

$$
J\left(x_{\alpha}, \dot{x}_{\alpha}\right)=-\frac{\alpha^{2}+(1-\alpha)^{2}}{2}
$$

which is maximal with $\alpha=1 / 2$ and $J\left(x_{1 / 2}, \dot{x}_{1 / 2}\right)=-1 / 4>-1 / 2$ which is better than the value of periodic problems (in fact, the q.p. with variable module of frequencies and a.p. problems have the only solution $(g, \dot{g})$ which gives 0$)$.

Before assuming the criterium to be concave, let us give a first result. In all this section, we set:

$$
J(x, u):=\mathcal{M}\left\{f_{0}(., x, u)\right\} .
$$

Remark 3.1. Denoting by $\operatorname{Val}(\mathbf{P})$ the value of a problem $(\mathbf{P})$, we have:

$$
\forall \tau>0, \quad \operatorname{Val}(\mathbf{A P P}) \geq \operatorname{Val}(\operatorname{VPPP}) \geq \operatorname{Val}(\mathbf{F P P P}(\tau)) .
$$

Proof. Indeed, we have for any $\tau>0$ :

$$
\mathbf{C}(\mathrm{APP}) \supset \mathbf{C}(\mathrm{VPPP}) \supset \mathbf{C}(\mathbf{F P P P}(\tau)) .
$$

Since the criteria are the same, the result is immediate. 
In all what follows in this section, we assume the two following conditions are satisfied:

- for all $t \in \mathbb{R}$, the function $f_{0}(t, .,$.$) defined on \mathbb{R}^{N} \times \mathbb{R}^{M}$ is concave;

- for all $t \in \mathbb{R}$, the function $f(t, . .$.$) is affine, i.e. there exists three matrix functions A, B$ and $b$ such that:

$$
\forall(t, x, u) \in \mathbb{R} \times \mathbb{R}^{N} \times \mathbb{R}^{M}, \quad f(t, x, u)=A(t) x+B(t) u+b(t) .
$$

Proposition 3.2. The set $\mathbf{S}(\mathbf{A P P})$ of solutions of (APP) is (strong and weak) closed and convex.

Proof. First, consider $\left(x_{1}, u_{1}\right),\left(x_{2}, u_{2}\right)$ admissible, and $\lambda \in[0 ; 1]$. Since $f_{0}(t, . .$.$) is concave, we have for all t$ :

$$
f_{0}\left(t, \lambda x_{1}(t)+(1-\lambda) x_{2}(t), \lambda u_{1}(t)+(1-\lambda) u_{2}(t)\right) \geq \lambda f_{0}\left(t, x_{1}(t), u_{1}(t)\right)+(1-\lambda) f\left(t, x_{2}(t), u_{2}(t)\right)
$$

and so by taking the mean, we have:

$$
J\left(\lambda x_{1}+(1-\lambda) x_{2}, \lambda u_{1}+(1-\lambda) u_{2}\right) \geq \lambda J\left(x_{1}, u_{1}\right)+(1-\lambda) J\left(x_{2}, u_{2}\right),
$$

so $J$ is concave, and since the state equation is affine, $\left(\lambda x_{1}+(1-\lambda) x_{2}, \lambda u_{1}+(1-\lambda) u_{2}\right)$ also satisfies this equation. So, we can say that the set $\mathbf{S}(\mathbf{A P P})$ is concave.

Now, consider a sequence $\left(x_{n}, u_{n}\right)_{n}$ of elements of S(APP) converging strongly or weakly to $(\bar{x}, \bar{u})$. Since the state equation is affine, it is a weak and strong continuous function and so $(\bar{x}, \bar{u})$ satisfies this equation. $J$ is continuous and concave, so it is also weakly usc. If convergence of $\left(x_{n}, u_{n}\right)_{n}$ is strong, we have immediately by continuity:

$$
J(\bar{x}, \bar{u})=\lim _{n \rightarrow+\infty} J\left(x_{n}, u_{n}\right)=\sup J
$$

and if the convergence is weak, we use the w-usc:

$$
J(\bar{x}, \bar{u}) \geq \lim _{n \rightarrow+\infty} J\left(x_{n}, u_{n}\right)=\sup J .
$$

In both cases, we see that $(\bar{x}, \bar{u})$ is a solution of the problem.

Remark 3.3. We can remark that:

(1) The fact that $\mathbf{S}(\mathbf{A P P})$ is strongly closed is dependent on the concavity of $f_{0}(t, . .$.$) .$

(2) The set of solutions of (VPPP) is not generally convex (for instance, when there exists two solutions whose ratio of periods is not rational). Here, we obtain a structure result which is not true for periodic problems with a variable period.

We now recall that if $x \in \mathrm{AP}^{0}\left(\mathbb{R}, \mathbb{R}^{N}\right)\left(\right.$ resp. $\left.\mathrm{B}^{2}\left(\mathbb{R}, \mathbb{R}^{N}\right)\right)$, and if $T>0$, we have the Besicovitch theorem: by denoting for all $\alpha \in \mathbb{R}, \tau_{\alpha} x:=x(.+\alpha)$, the sequence

$$
\left(\frac{1}{n} \sum_{j=0}^{n-1} \tau_{j T} x\right)_{n}
$$


converges in $\operatorname{AP}^{0}\left(\mathbb{R}, \mathbb{R}^{N}\right)$ (resp. $\mathrm{B}^{2}\left(\mathbb{R}, \mathbb{R}^{N}\right)$ ) to the $T$-periodification of $x, x^{T}$, which corresponds to the $T$ periodic part of the Fourier-Bohr expansion of $x$ :

$$
x \sim \sum_{\lambda \in \mathbb{R}} a_{\lambda} e_{\lambda} \Longrightarrow x^{T} \sim \sum_{\lambda \in \frac{2 \pi}{T} \mathbb{Z}} a_{\lambda} e_{\lambda} .
$$

The main lemma for all what follows is:

Lemma 3.4. Assume that for all $(x, u) \in \mathbb{R}^{N} \times \mathbb{R}^{M}$, the function $f_{0}(., x, u)$ is T-periodic. Then we have: $J\left(x^{T}, u^{T}\right) \geq J(x, u)$.

Proof. Because of the $T$-periodicity of the function $f_{0}$ with respect to $T$, we have for any $j \in \mathbb{Z}, J\left(\tau_{j T} x, \tau_{j T} u\right)=$ $J(x, u)$. We have by concavity of $J$ :

$$
J\left(\frac{1}{n} \sum_{j=0}^{n-1} \tau_{j T} x, \frac{1}{n} \sum_{j=0}^{n-1} \tau_{j T} u\right) \geq \frac{1}{n} \sum_{j=0}^{n-1} J\left(\tau_{j T} x, \tau_{j T} u\right)=J(x, u) .
$$

Now, by taking the limit as $n \rightarrow+\infty$ and by using the continuity of $J$, the preceding inequality shows that:

$$
J\left(x^{T}, u^{T}\right) \geq J(x, u) .
$$

Theorem 3.5. Assume that for all $(x, u) \in \mathbb{R}^{N} \times \mathbb{R}^{M}$, the function $f_{0}(., x, u)$ is T-periodic. Then we have:

(1) $\operatorname{Val}(\mathbf{A P P})=\operatorname{Val}(\mathbf{V P P P})=\operatorname{Val}(\mathbf{F P P P}(\mathbf{T}))$.

(2) Any solution of $(\mathbf{F P P P}(\mathbf{T}))$ is solution of the problems (VPPP), (APP).

(3) If $\mathbf{S}(\mathbf{A P P})$ is nonempty, it contains a T-periodic function (perhaps trivial), which is solution of the problems $(\mathbf{V P P P}),(\mathbf{F P P P}(\mathbf{T}))$.

Proof. Take arbitrary $(x, u)$. Since $\left(x^{T}, u^{T}\right)$ is $T$-periodic and since $J\left(x^{T}, u^{T}\right) \geq J(x, u)$ in view of the preceding lemma, we have:

$$
\operatorname{Val}(\operatorname{FPPP}(\mathrm{T})) \geq \operatorname{Val}(\mathrm{APP})
$$

Using Result 3.1, we deduce assertion (1). Now, if $(x, u)$ is a solution of $(\mathbf{F P P P}(\mathbf{T}))$, because of assertion (1), it is also a solution of problems (VPPP), (APP), so assertion (2) is justified. Assuming that $\mathbf{S}(\mathbf{A P P})$ is non empty, if $(x, u) \in \mathbf{S}(\mathbf{A P P})$, we have: $J\left(x^{T}, u^{T}\right) \geq J(x, u)$ so in fact $J\left(x^{T}, u^{T}\right)=J(x, u)$ and $\left(x^{T}, u^{T}\right)$ is a $T$-periodic solution of (APP) and it is so necessarily a solution to (VPPP), ( $\left.\mathbf{F P P P}(\mathbf{T})\right)$. This is assertion (3).

We study now the case when $f_{0}$ is autonomous. In this case, we can apply Theorem 3.5 with any $T$. It is here convenient to introduce a static optimization problem (SP):

$$
\left\{\begin{array}{l}
\text { Maximize } f_{0}(\bar{x}, \bar{u}) \\
(\bar{x}, \bar{u}) \in \mathbb{R}^{N} \times \mathbb{R}^{M} \\
\forall t \in \mathbb{R}, \quad A(t) \bar{x}+B(t) \bar{u}+b(t)=0 .
\end{array}\right.
$$


We obtain:

Theorem 3.6. We assume that $f_{0}$ is autonomous. Then we have for all $\tau>0$ :

(1) $\operatorname{Val}(\mathbf{A P P})=\operatorname{Val}(\mathbf{V P P P})=\operatorname{Val}(\mathbf{F P P P}(\tau))=\operatorname{Val}(\mathbf{S P})$.

(2) Any solution of one of the problems (APP), (VPPP), (FPPP $(\tau)),(\mathbf{S P})$ is solution of all the problems for which it is admissible.

(3) If $\mathbf{S}(\mathbf{A P P})$ is nonempty, it contains a constant function, which is solution of all the problems (VPPP), $(\operatorname{FPPP}(\tau)),(\mathbf{S P})$.

Proof. The main fact to use is that for any $(x, u)$ a.p., we can find $T>0$ such that $T \mathbb{Z} \cap \operatorname{Mod}(x, u)=\{0\}$. For such a $T$, we have $\left(x^{T}, u^{T}\right)=(\mathcal{M}\{x\}, \mathcal{M}\{u\})$ and by Lemma 3.4, we have $J(\mathcal{M}\{x\}, \mathcal{M}\{u\}) \geq J(x, u)$. So, $\operatorname{Val}(\mathbf{S P}) \geq \operatorname{Val}(\mathbf{A P P})$, and this implies the equalities of assertion (1). We deduce immediately as in Theorem 3.5 the second and the third assertions.

Remark 3.7. As a consequence, we see that if there exists $t_{0} \in \mathbb{R}$ such that:

$$
-b\left(t_{0}\right) \notin A\left(t_{0}\right) \mathbb{R}^{N}+B\left(t_{0}\right) \mathbb{R}^{M}
$$

then no problem has a solution.

Adaptation to locally compact abelian groups. In fact, the Besicovitch theorem on topological groups is true (see [3], Th. 3): given $\gamma_{0} \in \mathbb{G}$ and $x \in \mathrm{AP}^{0}\left(\mathbb{G}, \mathbb{R}^{N}\right)$ (resp. $\mathrm{L}^{2}\left(\mathbb{B} \mathbb{G}, \mathbb{R}^{N}\right)$ ), the sequence

$$
\left(\frac{1}{n} \sum_{j=0}^{n-1} \tau_{j \gamma_{0}} x\right)_{n}
$$

converges in $\operatorname{AP}^{0}\left(\mathbb{G}, \mathbb{R}^{N}\right)$ (resp. $\left.\mathrm{L}^{2}\left(\mathbb{B G}, \mathbb{R}^{N}\right)\right)$ to a function $x^{\gamma_{0}}$, which is $\gamma_{0}$-periodic, i.e. $\tau_{\gamma_{0}} x^{\gamma_{0}}=x^{\gamma_{0}}$. So, if we suppose that the operator $\Theta$ commutes with translations (i.e. $\tau_{\gamma} \circ \Theta=\Theta \circ \tau_{\gamma}$ ), the results of this section can be adapted to $\mathbb{G}$.

\section{NECESSARY CONDITIONS}

Let us define the Hamiltonian $H: \mathbb{B G} \times \mathbb{R}^{N} \times \mathbb{R}^{M} \times \mathbb{R} \times \mathbb{R}^{M} \rightarrow \mathbb{R}$ by the following formula:

$$
H\left(\gamma, x, u, \lambda_{0}, p\right):=\lambda_{0} f_{0}(\gamma, x, u)+p . f(\gamma, x, u) .
$$

We consider the problem (AAPP) only in the case when $(x, u)$ is varying in the set $\mathrm{D}^{2} \times K$, where $K$ is a non empty convex subset of $\mathrm{L}^{2}\left(\mathbb{B} \mathbb{G}, \mathbb{R}^{M}\right)$. In the first subsection, we study the case when $K=\mathrm{L}^{2}\left(\mathbb{B} \mathbb{G}, \mathbb{R}^{N}\right)$, and we study the general case in the second one.

\subsection{The case $K=\mathbf{L}^{2}\left(\mathbb{B} \mathbb{G}, \mathbb{R}^{M}\right)$}

We first give a weak Pontryagin principle with no qualification condition, and afterwards study the case when such a condition is satisfied.

Theorem 4.1 (weak Pontryagin principle). Let $(\bar{x}, \bar{u})$ be a solution of (AAPP). Then there exists $\left(\lambda_{0}, p\right) \in$ $\left(\mathbb{R} \times \mathrm{D}^{2}\right) \backslash\{0\}$ such that:

(1) $\Theta(\bar{x})=H_{p}\left(., \bar{x}, \bar{u}, \lambda_{0}, p\right)$;

(2) $\Theta^{*}(p)=H_{x}\left(., \bar{x}, \bar{u}, \lambda_{0}, p\right)$ ( $\Theta^{*}$ is the adjoint operator);

(3) $H_{u}\left(., \bar{x}, \bar{u}, \lambda_{0}, p\right)=0$. 
Proof. The first equation is in fact the state equation. Let us define the functionals $G: \mathrm{D}^{2} \times \mathrm{L}^{2}\left(\mathbb{B} G, \mathbb{R}^{M}\right) \rightarrow$ $\mathrm{L}^{2}\left(\mathbb{B G}, \mathbb{R}^{N}\right)$ and $J: \mathrm{D}^{2} \times \mathrm{L}^{2}\left(\mathbb{B} G, \mathbb{R}^{M}\right) \rightarrow \mathbb{R}$ by the following formulae:

$$
G(x, u):=f(., x, u)-\Theta(x)
$$

and

$$
J(x, u):=\int_{\mathbb{B G}} f_{0}(., x, u) \mathrm{d} \mu_{\mathbb{B G}} .
$$

We also define the following Nemytskii operators:

$$
\begin{aligned}
& \mathcal{N}_{f_{0}}: \mathrm{D}^{2} \times \mathrm{L}^{2}\left(\mathbb{B} \mathbb{G}, \mathbb{R}^{M}\right) \rightarrow \mathrm{L}^{2}(\mathbb{B} \mathbb{G}, \mathbb{R}), \quad \mathcal{N}_{f_{0}}(x, u):=f_{0}(., x, u), \\
& \mathcal{N}_{f}: \mathrm{D}^{2} \times \mathrm{L}^{2}\left(\mathbb{B} \mathbb{G}, \mathbb{R}^{M}\right) \rightarrow \mathrm{L}^{2}\left(\mathbb{B} \mathbb{G}, \mathbb{R}^{N}\right), \quad \mathcal{N}_{f}(x, u):=f(., x, u) .
\end{aligned}
$$

The results in [12] about continuity, Fréchet-differentiability and Gâteaux-differentiability of Nemytskii operators (Ths. 2.3, 2.6 and part a of the proof of Th. $\left.2.7^{1}\right)$ can also be adapted in the case when we are in $L^{p}(\mathbb{B} \mathbb{G}, Y)$. So, from the assumptions on $f, f_{0}$, we know immediately that:

- $\mathcal{N}_{f_{0}}$ is Fréchet-differentiable (written F-differentiable), and its F-differential is:

$$
\mathcal{N}_{f_{0}}^{\prime}(x, u) \cdot(h, k)=D_{x} f_{0}(., x, u) \cdot h+D_{u} f_{0}(., x, u) \cdot k .
$$

- $\mathcal{N}_{f}$ is Gâteaux-differentiable (written G-differentiable), and its G-differential is:

$$
D^{G} \mathcal{N}_{f}(x, u) \cdot(h, k)=D_{x}^{G} f(., x, u) \cdot h+D_{u}^{G} f(., x, u) \cdot k .
$$

- When $(h, k)$ is fixed, the operator:

$$
(x, u) \rightarrow D_{x}^{G} f(., x, u) \cdot h+D_{u}^{G} f(., x, u) . k
$$

is continuous (we will say that the functional is continuously G-differentiable as in [1], p. 88).

From that, we deduce by superposition that the functional $G$ (resp. $J$ ) is well defined and F-differentiable (resp. continuously G-differentiable) from $\mathrm{D}^{2} \times \mathrm{L}^{2}\left(\mathbb{B} G, \mathbb{R}^{N}\right)$ to $\mathrm{L}^{2}\left(\mathbb{B} \mathbb{G}, \mathbb{R}^{N}\right)$ (resp. $\left.\mathbb{R}\right)$. Moreover, the differentials are given by:

$$
\begin{aligned}
& J^{\prime}(x, u) \cdot(h, k)=\int_{\mathbb{B G}}\left(D_{x} f_{0}(., x, u) \cdot h+D_{u} f_{0}(., x, u) \cdot k\right) \mathrm{d} \mu_{\mathbb{B} \mathbb{G}} \\
& D^{G} G(x, u) .(h, k)=D_{x}^{G} f(., x, u) \cdot h+D_{u}^{G} f(., x, u) \cdot k-\Theta(h) .
\end{aligned}
$$

Our problem is to maximise $J(x, u)$ under the constraint $G(x, u)=0$. The Lagrange multiplier rule is also valid since because of the continuity of G-differential of the constraint, the Clarke generalized gradient is reduced to this G-differential and we can use the Clarke version of this theorem ([1], Props. 6.10 and 6.15). So, the first order condition is:

$$
\exists\left(\lambda_{0}, \Lambda\right) \in\left(\mathbb{R} \times \mathrm{L}^{2}\left(\mathbb{B} G, \mathbb{R}^{M}\right)^{\prime}\right) \backslash\{0\}, \quad \lambda_{0} J^{\prime}(\bar{x}, \bar{u})+\Lambda \circ G^{\prime}(\bar{x}, \bar{u})=0 .
$$

Since $\mathrm{L}^{2}\left(\mathbb{B} G, \mathbb{R}^{M}\right)$ is a Hilbert space, we can identify it with its dual space. Namely, one can find $p \in \mathrm{L}^{2}\left(\mathbb{B} \mathbb{G}, \mathbb{R}^{M}\right)$ such that for any $\zeta \in \mathrm{L}^{2}\left(\mathbb{B} \mathbb{G}, \mathbb{R}^{M}\right)$ :

$$
\Lambda(\zeta)=\int_{\mathbb{B G}} p(\gamma) \cdot \zeta(\gamma) \mathrm{d} \mu_{\mathbb{B} \mathbb{G}}(\gamma) .
$$

\footnotetext{
${ }^{1}$ The part a of the proof show that the Nemytskii operator is Gâteaux-differentiable in this case.
} 
Moreover, $\left(\lambda_{0}, \Lambda\right) \neq 0$ implies $\left(\lambda_{0}, p\right) \neq 0$. We recall that the differentials of $J$ and $G$ are given by:

$$
\begin{gathered}
J^{\prime}(x, u) \cdot(h, k)=\mathcal{M}\left\{D_{x} f_{0}(., x, u) \cdot h+D_{u} f_{0}(., x, u) k\right\} \\
G^{\prime}(x, u) .(h, k)=D_{x} f(., x, u) . h+D_{u} f(., x, u) . k-\Theta(h) .
\end{gathered}
$$

Replacing this in the necessary condition, and we obtain for all $(h, k) \in \mathrm{D}^{2} \times \mathrm{L}^{2}\left(\mathbb{B} \mathbb{G}, \mathbb{R}^{M}\right)$ :

$$
\int_{\mathbb{B G}}\left[\left(\lambda_{0} D_{x} f_{0}(., \bar{x}, \bar{u})+p \cdot\left(D_{x} f(., \bar{x}, \bar{u})\right)\right) \cdot h+\left(\lambda_{0} D_{u} f_{0}(., \bar{x}, \bar{u})+p \cdot\left(D_{u} f(., \bar{x}, \bar{u})\right)\right) \cdot k-p \cdot \Theta(h)\right] \mathrm{d} \mu_{\mathbb{B} \mathbb{G}}=0 .
$$

By definition of the adjoint operator, we have:

$$
\int_{\mathbb{B} \mathbb{G}} p \cdot \Theta(h) \mathrm{d} \mu_{\mathbb{B} \mathbb{G}}=\langle p ; \Theta(h)\rangle_{\mathrm{L}^{2}\left(\mathbb{B} \mathbb{G}, \mathbb{R}^{M}\right)}=\left\langle\Theta^{*}(p) ; h\right\rangle_{\mathrm{L}^{2}\left(\mathbb{B} \mathbb{G}, \mathbb{R}^{M}\right)}=\int_{\mathbb{B} \mathbb{G}} \Theta^{*}(p) . h \mathrm{~d} \mu_{\mathbb{B} \mathbb{G}} .
$$

By replacing this on the necessary condition, we obtain for all $(h, k) \in \mathrm{D}^{2} \times \mathrm{L}^{2}\left(\mathbb{B} \mathbb{G}, \mathbb{R}^{M}\right)$ :

$$
\int_{\mathbb{B} \mathbb{G}}\left[\left(\lambda_{0} D_{x} f_{0}(., \bar{x}, \bar{u})+p \cdot\left(D_{x} f(., \bar{x}, \bar{u})-\Theta^{*}(p)\right)\right) \cdot h+\left(\lambda_{0} D_{u} f_{0}(., \bar{x}, \bar{u})+p \cdot\left(D_{u} f(., \bar{x}, \bar{u})\right)\right) \cdot k\right] \mathrm{d} \mu_{\mathbb{B} \mathbb{G}}=0
$$

which gives:

$$
\Theta^{*}(p)=\lambda_{0} D_{x} f_{0}(., \bar{x}, \bar{u})+p .\left(D_{x} f(., \bar{x}, \bar{u})\right)
$$

and

$$
\lambda_{0} D_{u} f_{0}(., \bar{x}, \bar{u})+p \cdot\left(D_{u} f(., \bar{x}, \bar{u})\right)=0
$$

i.e.:

$$
\Theta^{*}(p)=H_{x}\left(., \bar{x}, \bar{u}, \lambda_{0}, p\right), \text { and } H_{u}\left(., \bar{x}, \bar{u}, \lambda_{0}, p\right)=0 .
$$

We next give some conditions which allow us to take $\lambda_{0}=1$. To state the condition (C3) below, we introduce some notations. We say that the operator $\Theta$ diagonalize the Fourier transform if there exists a function $\psi:(\mathbb{B} G)^{\prime} \rightarrow \mathbb{C}$ such that:

$$
\forall u \in \mathrm{D}^{2}, \quad \forall \chi \in(\mathbb{B G})^{\prime}, \quad \widehat{\Theta u}(\chi)=\psi(\chi) \hat{u}(\chi) .
$$

This is the case for our three particular operators on $\mathbb{R}\left(\right.$ resp. $\mathbb{T}^{m}$, resp. $\left.\mathbb{Z}\right)$, where we have $\psi(\lambda):=i \lambda$ for any $\lambda \in \mathbb{R}\left(\right.$ resp. $\psi(\nu):=i \nu . \omega$ for any $\nu \in \mathbb{Z}^{m}$, resp. $\psi(\lambda):=\exp (i \lambda)$ for any $\left.\lambda \in[0 ; 1)\right)$.

Proposition 4.2. Moreover, if one of these conditions is satisfied, we can take $\lambda_{0}=1$ in the preceding theorem.

(C1) The mapping $(h, k) \mapsto A() h+.B() k-.\Theta(h)$ is onto from $\mathrm{D}^{2} \times \mathrm{L}^{2}\left(\mathbb{B G}, \mathbb{R}^{M}\right)$ to $\mathrm{L}^{2}\left(\mathbb{B} G, \mathbb{R}^{N}\right)$.

(C2) For all $\zeta \in \mathrm{L}^{2}\left(\mathbb{B} \mathbb{G}, \mathbb{R}^{N}\right)$, there exists $h \in \mathrm{D}^{2}$ such that:

$$
-\Theta(h)+A(.) h=\zeta .
$$

(C3) $\Theta$ diagonalize the Fourier transform, A is constant (with set of eigenvalues denoted by $S p(A)$ ) and:

$$
\inf _{(\sigma, \chi) \in S p(A) \times(\mathbb{B G})^{\prime}}|\sigma-\psi(\chi)|>0 .
$$

Proof. Since $G^{\prime}(\bar{x}, \bar{u}) \cdot(h, k)=A() . h+.B(.) \cdot k-\nabla h$, condition $(\mathbf{C} 1)$ is the standard one. Let us show that:

$$
(\mathrm{C} 3) \Rightarrow(\mathrm{C} 2) \Rightarrow(\mathrm{C} 1)
$$

This will give the result. 
$(\mathbf{C 3}) \Rightarrow(\mathbf{C 2})$. By taking a trigonal form of $A$, we see that it is sufficient to make the proof when $N=1$. We wish to solve:

$$
-\Theta(h)+\alpha h \sim_{2} \zeta
$$

We take the Fourier transform of this expression. This gives:

$$
\forall \chi \in(\mathbb{B} G)^{\prime}, \quad(\alpha-\psi(\chi)) \hat{h}(\chi)=\zeta(\chi) .
$$

Since $\alpha-\psi(\chi)$ is never 0 , this defines uniquely the Fourier transform of $h$. This expression is a Fourier transform of a function in $\mathrm{D}^{2}$ if and only if:

$$
\int_{(\mathbb{B} \mathbb{G})^{\prime}}\left(1+|\psi(\chi)|^{2}\right)|\hat{h}(\chi)|^{2} \mathrm{~d} \mu(\chi)<+\infty
$$

But this integral can be written:

$$
\int_{(\mathbb{B} \mathbb{G})^{\prime}} \Psi(\psi(\chi))|\hat{\zeta}(\chi)|^{2} \mathrm{~d} \mu(\chi)
$$

where the function $\Psi: \mathbb{C} \rightarrow \mathbb{R}$ is defined by: $\Psi(z):=\frac{1+|z|^{2}}{|\alpha-z|^{2}}$. The function $\Psi$ is clearly bounded on $\mathbb{C}$, and so our integral is less or equal to:

$$
\|\Psi\|_{\infty}^{2}\|\zeta\|_{2}^{2}<+\infty
$$

and this gives (C2).

$(\mathbf{C 2}) \Rightarrow(\mathbf{C 1})$. It is sufficient to take $(h, 0)$.

Remark 4.3. When $\mathbb{G}=\mathbb{R}$ or $\mathbb{T}^{m}$, the last assumption of (C3) is equivalent to the fact that $A$ has no pure imaginary eigenvalue and when $\mathbb{G}=\mathbb{Z}$, this is equivalent to the fact that $A$ has no eigenvalue of modulus 1 .

\subsection{Case when $\boldsymbol{K}$ is convex}

In all this subsection, we assume that the qualification condition (C1) is satisfied, and we also suppose that:

$$
\exists(\hat{x}, \hat{u}) \in \mathrm{D}^{2} \times \operatorname{Int} K, \quad A . \hat{x}+B . \hat{u}+b=0 .
$$

Theorem 4.4 (weak Pontryagin principle). Under these assumptions, if $(\bar{x}, \bar{u})$ is an optimal solution, then there exists $p \in \mathrm{D}^{2} \backslash\{0\}$ such that:

(1) $\Theta(\bar{x})=H_{p}(., \bar{x}, \bar{u}, 1, p)$;

(2) $\Theta^{*}(p)=-H_{x}(., \bar{x}, \bar{u}, 1, p)$;

(3) $H_{u}(., \bar{x}, \bar{u}, 1, p) \in N_{K}(\bar{u})$,

where $N_{K}(\bar{u})$ is the normal cone to $K$ at $\bar{u}$.

Proof. We again take the notations of the proof of Theorem 4.1. Because of the new assumption, we know that

$$
0 \in \operatorname{Int}\left(\left(\mathrm{B}^{1,2} \times K\right)-G^{-1}(0)\right) .
$$

Indeed, there exists $r>0$ such that if $\|\delta u\|_{\mathrm{L}^{2}\left(\mathbb{B} \mathbb{G}, \mathbb{R}^{M}\right)}<r$, we have $\hat{u}+\delta u \in K$ and so, for any $\delta x$, we have:

$$
(\delta x, \delta u)=(\hat{x}+\delta x, \hat{u}+\delta u)-(\hat{x}, \hat{u}) \in\left(\mathrm{B}^{1,2} \times K\right)-G^{-1}(0)
$$

In view of $[1]((51)$, p. 72$)$, we obtain:

$$
N_{\left(\mathrm{B}^{1,2} \times K\right) \cap G^{-1}(0)}=N_{\left(\mathrm{B}^{1,2} \times K\right)}+N_{G^{-1}(0)} .
$$


But:

and with (C1) this gives:

$$
N_{\left(\mathrm{B}^{1,2} \times K\right)}=N_{\mathrm{B}^{1,2}} \times N_{K}=\{0\} \times N_{K}
$$

$$
N_{G^{-1}(0)}(\bar{x}, \bar{u})=\operatorname{Ker} G^{\prime}(\bar{x}, \bar{u}) .
$$

The necessary first order condition for this problem is:

$$
D J(\bar{x}, \bar{u}) \in N_{\left(\mathrm{B}^{1,2} \times K\right) \cap G^{-1}(0)}(\bar{x}, \bar{u})
$$

from what we deduce:

$$
D J(\bar{x}, \bar{u}) \in N_{\mathrm{B}^{1,2} \times K}(\bar{x}, \bar{u})+N_{G^{-1}(0)}(\bar{x}, \bar{u})
$$

so there exists $\Lambda \in\left(\mathrm{B}^{2}\left(\mathbb{R}, \mathbb{R}^{M}\right)\right)^{\prime}$ such that:

$$
D J(\bar{x}, \bar{u})+\Lambda \circ D G(\bar{x}, \bar{u}) \in\{0\} \times N_{K}(\bar{u})
$$

and by reasoning as in the proof of Theorem 4.1, we obtain the result.

Remark 4.5. The two theorems about first order necessary conditions, 4.1 and 4.4, assert the existence of an adjoint variable $p$ which is a.p. This property of the adjoint arc can be interpreted as a condition corresponding to the transversality condition in the classical Pontryagin Maximum Principle.

\section{An existence Result}

We will now give an existence result. We again look for $(x, u)$ in $\mathrm{D}^{2} \times K$, where $K$ is now a weakly closed subset of $\mathrm{L}^{2}\left(\mathbb{B} \mathbb{G}, \mathbb{R}^{M}\right)$.

Theorem 5.1. We assume that $f_{0}(t, .$.$) is concave for all t \in \mathbb{B} \mathbb{G}$ and that there exists $\alpha \in L^{1}(\mathbb{B} \mathbb{G}, \mathbb{R})$ and $\beta \in \mathbb{R}_{*}^{+}$such that:

$$
\forall(t, x, u) \in \mathbb{B} G \times \mathbb{R}^{N} \times \mathbb{R}^{M}, \quad f_{0}(t, x, u) \leq \alpha(t)-\beta\left(|x|^{2}+|u|^{2}\right) .
$$

The problem (AAPP) has at least a solution.

Proof. We set $\Gamma=G^{-1}(0)$. We have to maximize $J$ on $\Gamma$ which is strongly and weakly closed since $G$ is affine and continuous. We set $S:=\sup _{(x, u) \in \mathrm{D}^{2} \times \mathrm{L}^{2}\left(\mathbb{B} \mathbb{G}, \mathbb{R}^{M}\right)} J(x, u)$. Clearly, $S \in \mathbb{R}$ since for all $(x, u) \in \mathrm{D}^{2} \times \mathrm{L}^{2}\left(\mathbb{B} \mathbb{G}, \mathbb{R}^{M}\right)$, we have:

$$
J(x, u) \leq \int_{\mathbb{B} \mathbb{G}} \alpha \mathrm{d} \mu_{\mathbb{B} \mathbb{G}} .
$$

Let $\left(x_{n}, u_{n}\right)_{n}$ be a sequence which satisfies the two following conditions:

- $\forall n,\left(x_{n}, u_{n}\right) \in \Gamma \cap K$

- $J\left(x_{n}, u_{n}\right) \geq S-1 / n$.

Let us first show that the sequence $\left(N_{n}\right)_{n}$ with $N_{n}:=\left\|x_{n}\right\|_{\mathrm{D}^{2}}^{2}+\left\|u_{n}\right\|_{\mathrm{L}^{2}\left(\mathbb{B G}, \mathbb{R}^{M}\right)}^{2}$ is bounded. Indeed, since:

$$
S-1 / n \leq J\left(x_{n}, u_{n}\right) \leq \int_{\mathbb{B} \mathbb{G}} \alpha \mathrm{d} \mu_{\mathbb{B} \mathbb{G}}-\beta\left(\left\|x_{n}\right\|_{\mathrm{L}^{2}\left(\mathbb{B} \mathbb{G}, \mathbb{R}^{N}\right)}^{2}+\left\|u_{n}\right\|_{\mathrm{L}^{2}\left(\mathbb{B G}, \mathbb{R}^{M}\right)}^{2}\right)
$$

we obtain immediately:

$$
\left\|x_{n}\right\|_{\mathrm{L}^{2}\left(\mathbb{B} \mathbb{G}, \mathbb{R}^{N}\right)}^{2}+\left\|u_{n}\right\|_{\mathrm{L}^{2}\left(\mathbb{B} \mathbb{G}, \mathbb{R}^{M}\right)}^{2} \leq C_{1}:=\frac{1}{\beta}\left[\int_{\mathbb{B} \mathbb{G}} \alpha \mathrm{d} \mu_{\mathbb{B} \mathbb{G}}-(S-1)\right]
$$


Moreover, from $\Theta\left(x_{n}\right)=A . x_{n}+B . u_{n}+b$, we obtain:

$$
\begin{gathered}
\left\|\Theta\left(x_{n}\right)\right\|^{2} \leq 4\left(\|A\|_{\infty}\left\|x_{n}\right\|_{L^{2}\left(\mathbb{B G}, \mathbb{R}^{N}\right)}^{2}+\|B\|_{\infty}\left\|u_{n}\right\|_{\mathrm{L}^{2}\left(\mathbb{B} \mathbb{G}, \mathbb{R}^{M}\right)}^{2}+\|b\|_{\mathrm{L}^{2}(\mathbb{B} \mathbb{G}, \mathbb{R})}^{2}\right) \leq \\
C_{2}:=4\left(\|b\|_{\mathrm{L}^{2}(\mathbb{B} \mathbb{G}, \mathbb{R})}^{2}+C_{1} \max \left\{\|A\|_{\infty} ;\|B\|_{\infty}\right\}\right) .
\end{gathered}
$$

So, finally we have: $N_{n} \leq C_{1}+C_{2}$. Since the sequence $\left(x_{n}, u_{n}\right)_{n}$ is bounded, it has a subsequence weakly convergent to $(\bar{x}, \bar{u})$. Since $G$ is affine, it is weakly continuous and so, for all $n,\left(x_{n}, u_{n}\right) \in G^{-1}(0)$, we have $(\bar{x}, \bar{u}) \in G^{-1}(0)$. Since $K$ is weakly closed, we also have $(\bar{x}, \bar{u}) \in G^{-1}(0)$. Since $J$ is concave, it is weakly u.s.c. and so we obtain $J(\bar{x}, \bar{u}) \geq S$, and so $J(\bar{x}, \bar{u})=S$.

\section{REFERENCES}

[1] J.-P. Aubin, Optima and Equilibria: an introduction to Nonlinear Analysis. Springer, 2nd Edn. (1988).

[2] A.S. Besicovitch, Almost Periodic Functions. Cambridge University Press, Cambridge (1932) (and Dover, 1954).

[3] J. Blot, Le théorème de Markov-Kakutani et la presque-périodicité, Fixed Point Theory and Applications, M. Théra and J.B. Baillon Eds., Pitman Research Notes in Mathematical Series 252, Longman, London (1991) 45-56.

[4] J. Blot, Oscillations presque-périodiques forcées d'équations d'Euler-Lagrange. Bull. Soc. Math. France 122 (1994) $285-304$.

[5] J. Blot, Variational Methods for the Almost Periodic Lagrangian Oscillations. Preprint, Cahiers Eco et Maths No. 96.44 (1996).

[6] J. Blot and D. Pennequin, Spaces of quasi-periodic functions and oscillations in dynamical systems. Acta Appl. Math. 65 (2001) 83-113.

[7] J. Blot and D. Pennequin, Existence and structure results on Almost Periodic solutions of Difference Equations. J. Diff. Equa. Appl. 7 (2001) 383-402.

[8] H. Bohr, Almost Periodic Functions. Julius Springer, Berlin (1933) (Chelsea Publishing Company, N.Y., 1947).

[9] F. Colonius, Optimal Periodic Control, in Lect. Notes Math. 1313, Springer, Berlin (1988).

[10] C. Corduneanu, Almost Periodic Functions. Chelsea (1989).

[11] G. Da Prato and A. Ichikawa, Optimal control of linear systems with a.p. inputs. SIAM J. Control Optim. 25 (1987) $1007-1019$.

[12] D.G. De Figueiredo, Lectures on the Ekeland Variational Principle with Applications and Detours. Tata Institute of Fundamental Research, Bombay (1989).

[13] J. Favard, Leçons sur les fonctions presque-périodiques. Gauthiers-Villars, Paris (1933).

[14] A. Halanay, Optimal Control of Periodic solutions. Rev. Rouman. Mat. Pure Appl. 19 (1974) 3-16.

[15] V.P. Havin and N.K. Nikolski Eds., Commutative Harmonic Analysis II. Springer, Berlin (1991).

[16] E. Hewitt, K.A. Ross, Abstract Harmonic Analysis I $\&$ II. Springer, Berlin, 2nd Edn. (1979) (and 1970).

[17] F.J.M. Horn and J.E. Bailey, An application of the theorem of relaxed control to the problem of increasing catalyst selectivity. J. Opt. Theory Appl. 2 (1968) 441-449.

[18] A. Kovaleva, Optimal Control of Mechanical Oscillations. Springer, Berlin (1999).

[19] J.L. Mauclaire, Intégration et Théorie des Nombres. Travaux en Cours, Hermann, Paris (1986).

[20] G.M. N'Guérékata, Almost automorphic and almost periodic functions in abstract spaces. Kluwer Academic Publishers (2001)

[21] P. Nistri, Periodic Control Problems for a class of nonlinear periodic differential systems. Nonlinear Anal. Theor. Meth. Appl. 7 (1983) 79-90.

[22] D. Pennequin, Existence results on almost periodic solutions of discrete time equations. Discrete Cont. Dynam. Syst. 7 (2001) $51-60$.

[23] I.C. Percival, Variational principles for the invariant toroids of classical dynamics. J. Phys. A: Math. Nucl. Gen. 7 (1974) 794-802.

[24] I.C. Percival, Variational principles for invariant tori and cantori. A.I.P. Conf. Proc. 57 (1979) 302-310.

[25] L. Pontryagin, Topological Groups. N.Y. Gordon and Breach (1966).

[26] J.L. Speyer, Periodic optimal flight. J. Guid. Control Dynam. 61 (1996) 745-754.

[27] W. Rudin, Fourier Analysis on Groups. Interscience Publishers, N.Y. (1962).

[28] A. Weil, L'intégration dans les Groupes Topologiques. Hermann, Paris (1940). 